\title{
Complications following T-tube and C-tube Drainage of the Common Bile Duct
}

\author{
Rovena Bode ${ }^{1 *}$, Klevis Shella ${ }^{2}$, Hysni Dede ${ }^{2}$, Xheladin Draçini ${ }^{1}$, Etmond Çeliku ${ }^{1}$
}

Received: 25 March 2021 / Accepted: 19 April 2021 / Published online: 20 July 2021

This article is published with open access at $\mathbf{h t t p s : / / j o u r n a l . a s t e s . o r g . a l}$

(C) The author(s) 2021. \& Copyright (C) 2021, the Albanian Society for Trauma and Emergency Surgery

(c) The Albanian Journal of Trauma and Emergency Surgery is an Open Access Journal. All articles are distributed under the terms of the Creative Commons Attribution Non-Commercial License: http://creativecommons.org/licenses/by-nc/4.0/) which permits unrestricted non-commercial use, distribution, and reproduction in any medium provided the original work is properly cited.

\section{Abstract}

The aim of this study is to assess the complications of T-tube (Kehr) and C- tube (Cystic) drainage used for biliary drainage, following biliary surgery.

We evaluate all possible complications, related to the tube in situ, during cholangiography and following t- tube removal retrospectively, during a 4- year period 2016-2019.

T-tubes were inserted in 48 patients, with 11 (22.8\%) patients experiencing complications related to T-tube. A broad spectrum of complications was found, ranging from biliary-specific complications such as a biliary leak, biliary peritonitis, and retained stones, to systemic general complications as wound infection, pulmonary thromboembolism, and internal hemorrhage.

Although this retrospective study has underestimated the incidence of T-tube complications, it has demonstrated significant morbidity related to T-tube use, which poses the need for replacement by minimally invasive surgical techniques.

Key words: choledochotomy, T-tube, C-tube, CBD exploration, ES, ERCP

\section{Introduction:}

T- tube placement into the common bile duct is most commonly performed after common bile duct (CBD) exploration for cholelithiasis or repair of an iatrogenic CBD injury. T-tube drainage following choledochotomy has been a standard procedure in the era of open cholecystectomy (1), although controversies as to whether it is the best procedure [2, 3]. There are different methods for extracting stones from the common bile duct and endoscopic

Original article, no submission or publication in advance or in parallel

* Corresponding author:

Rovena Bode MD, MSc, PhD,

$\triangle$ rovenabode@gmail.com

1 Department of General Surgery, University Hospital Center," Mother Teresa" Tirana, ALBANIA

2 Faculty of Medicine, University of Medicine of Tirana, ALBANIA extraction is the commonly procedure today $[4,5]$. Management of choledocholithiasis has changed radically in recent years following innovation and developments in minimally invasive surgical techniques. Endoscopic Retrograde Cholangiopancreatography (ERCP) and endoscopic sphincterotomy (ES) followed by laparoscopic cholecystectomy are a popular option for this disease [6]. Because of the T- tube related morbidity, prolonged hospital stays and higher hospital expenses, authors have described various ways to decompress the biliary tree [7, 8]. Consequently, some surgeons have recommended primary closure of the common bile duct immediately after choledochotomy to reduce the risk of T-tube-related complications, and the use of modified biliary stents [9].

And yet, there are still a few indications for choledochotomy and T- tube placement nowadays.

T-tube functioning is based on the formation of multiple fibrous adhesions closing off any significant defect [3]. T tube allows passive decompression for the biliary tree [4], postoperative percutaneous access to the CBD via $\mathrm{T}$ tube cholangiography [5], and extraction of calculi via a 
mature drain tract. It is a necessary instrument in the biliary surgery to prevent bile leakage from choledochotomy, prevent postoperative stricture and promotes the formation of the fistulous tract. Postoperative morbidity after bile duct exploration with $\mathrm{T}$ tube insertion is mainly related to tube removal and incomplete sinus tract formation leading to serious complications.

\section{Materials and methods:}

All patients undergoing supraduodenal choledochotomy and T/C-tube drainage during the period January 2016 December 2019 were entered into the study (retrospective analysis).

We should underline that a part of patients was operated in the emergency surgical unit, for cholecystitis, for biliary fistula, for traumatic injury on the biliary tree following laparoscopic or open cholecystectomy, for liver abscesses... where laparoscopic cholecystectomy, either ERCP and endoscopic sphincterotomy could not be done. The other cases as elective operations, when ERCP and laparoscopic cholecystectomy encountered objective difficulties and contraindications.

These patients had all received a single intravenous injection of cefazoline $1 \mathrm{gr}$, one hour preoperatively with the premedication. A culture was obtained from the common bile duct at the time of operation only in a part of patients. An appropriate size latex T-tube (12-20 Fr) was inserted on completion of exploration and the duct was closed on running or interrupted absorbable sutures. An abdominal drain was placed in subhepatic space. The postoperative management of $\mathrm{T}$ tube was focused on:

o Drainage of bile through T-tube up to 7-th post-operative day (POD)

o Progressive clamping of T-tube from $8^{\text {th }}$ POD and observation of the patient for pain, fever and jaundice

o T-tube removal was preceded by a control colangiography between 7-30 days.

o T-tube removal time in our practice has varied from 8 days minimum to 4 months in special cases.

o If there was no residual calculus and free flow into the duodenum, the tube was immediately removed.

o Contraindication of removal: Charcot's triad, pain or leakage after clamping and abnormal T-tube cholangiogram

o Time between colangiography and T-tube removal in our series varied from 12 to 48 hours.

\section{Results:}

The study involved 48 patients $(11 \mathrm{~m}-37 \mathrm{f})$, with a total of 50 T- tubes and 2 C-tubes. External biliary drainage through T-tube was done in 46 of them with 48 T-tubes. In 2 patients a $\mathrm{C}$-tube to decompress the biliary tree was placed.

34 explorations were for duct stones at the time of cholecystectomy, for hydatid cysts and liver abscesses.
11 explorations were for treating complications of previous biliary surgery such as residual duct stones from previous operations, dislodgement of $\mathrm{T}$ tubes, biblioenteric anastomotic leaks and iatrogenic injuries. Three explorations were for protecting biliobiliary and bilioenteric anastomosis. Two tubes were reinserted after dislodgment from biliary tree.

- T-tube and C-tube drainage of biliary tree were performed mainly for cholelithiasis, hydatid cysts of liver and liver abscesses (34 patients, 34 T-tubes and 2 C-tubes).

o In 28 of them a supraduodenal choledochotomy and a T-tube drainage was done after open common bile duct exploration at the time of cholecystectomy

o In two of them a trans cystic biliary decompression (C-tube drainage) was performed following open cholecystectomy.

o In 3 other cases a common bile duct drainage was performed because of hydatid cysts of the liver

o In 3 other patients the common bile duct drainage was necessary because of liver abscesses.

- T- tube was performed also for the treatment of complications of previous biliary surgery (11 patients, 13 T-tubes).

o In 4 patients for residual common bile duct stones left after cholecystectomy (one open and three laparoscopic),

o In one case for an early partial dislodgment (one limb of the T-tube), $\mathrm{T}$ tube was reinserted

o In one case for a complete dislodgment of $\mathrm{T}$ tube, $\mathrm{T}$ tube was reinserted

o In 2 other cases for bilioenteric anastomotic leak and

o In 3 patients for common bile duct iatrogenic injury after laparoscopic cholecystectomy (2) and liver trauma (1), presented with biliary fistula or obstructive jaundice.

- T-tube was performed to protect biliobiliary anastomosis (one case) and bilioenteric anastomosis (2 cases) in pancreaticoduodenectomy (a total of 3 patients, 3 T-tubes).

T-tubes were inserted in 48 patients, with $11(22.8 \%)$ patients experiencing a total of 32 complications related to T-tube use.

- Complications occurring with the T/C-tube in situ $[7(14.6 \%)$ patients, more than one complication was found in one patient]

o Fluid and electrolytic imbalance was found in 4 patients $(8.3 \%)$. Because of the difficulties in laboratory electrolytic exams, the defining criteria were partially laboratory, blood gas analysis and clinical also. All patients were treated conservatively.

o Bacteremia and ascendent cholangitis was found in $2(4 \%)$ patients. Because of the impossibility of bile culture in every patient, defining criteria were partially clinical (Charcot's triad) and apart laboratoric. They all were treated conservatively.

o Early dislodgement of T- tube, (a limb of T-tube) 
was found in one $(2 \%)$ patient. The patient required reoperation and reinsertion of T-tube.

o T-tube dislocation with biliary peritonitis, in one ( $2 \%$ ) patient. The patient was reoperated.

o T-tube wound infection in $3(6.25 \%)$ patients, all treated conservatively.

o Biliary leak around the $T$ - tube in $2(4.1 \%)$ patients, treated conservatively.

o Hemoperitoneum in $1(2 \%)$ patient, reoperated.

o Pulmonary thromboembolism in one (2\%) patient

- Complications during cholangiography [6(12.5\%) patients, more than one complication were found in one patient] During cholangiography through T/C-tube we found.

o Residual stone (filling defect), in 4(8.3\%) patients, t-tube was left for more than 3 weeks, required endoscopic removal.

o Bacteremia in $5(10.67 \%)$ patients, treated conservatively.

o Biliary leakage in $2(4.1 \%)$ patients (peritubular spillage), treated conservatively

- Complications following T/C-tube removal [8(16.67\%) patients].

o In one (2.1\%) patient transient fever and abdominal pain, required strong analgesia and antibiotics

o In $2(4.1 \%)$ patients' biliary leakage, developed the clinical picture of localized peritonitis, treated by ultrasound guided percutaneous drainage

o In $2(4.1 \%)$ patients' biliary peritonitis, reoperated and reinsertion of $\mathrm{T}$ tube

o In $2(4.1 \%)$ patients prolonged external biliary fistula

o In one $(2.1 \%)$ patient intraperitoneal collections, reoperated

\section{Discussion:}

T-tube drainage has been the standard method of management after supraduodenal choledochotomy and not only [3]. As we see the spectrum of its use was wide. The rationale for its use is that it minimises the risk of leakage in the early postoperative period if there is persistent distal duct obstruction. It also allows further radiological examination and may facilitate removal of residual calculi. However, it was associated with biliary specific complications and general systemic complications.

As seen, in two patients, the transcystic approach was preferred. Whenever feasible, the trans-cystic duct approach is the preferred technique, because it is less invasive and has proved to be safe and efficient $[11,12]$. The indications for trans-cystic CBDE, however, are limited to stones that are smaller than the size of the cystic duct, to a number of stones -less than five, to stones located in the lower CBD and not higher up in the common hepatic duct, and when a favorable anatomy of the cystic duct-CBD junction is present [13].

A latex T-tube is the most suitable type for short term biliary drainage [10]. It is said to produce a rapid intraperitoneal reaction with good track formation, thus biliary leakage should be unlikely. The risk of bile leakage after elective removal of T-tube from the common bile duct varies between 0,8 and $5 \%[14,15]$. It seems that bile leakage after removal of the T-tube may be caused by several factors including poor operative technique (passing of sutures through the tube while narrowing the hole of the bile duct around the tube), extremely big diameter of the tube etc. It may result also from sites other than the choledochotomy e.g. iatrogenic lesion of the common bile duct. The presence of increased pressure within the common bile duct caused by spasm of the sphincter of Oddi or retained calculi may increase the leakage [16].

However, in 7 cases of this study there was leakage into the peritoneal cavity from the choledochotomy site after removal of the T-tube. The reasons for leakage are unclear, though poor intraperitoneal reaction and duct trauma on removal are possible factors. Nevertheless, biliary leakage is a potentially life threatening complication and at the very least will delay discharge from hospital. Infact two patients who experienced biliary leak, developed the clinical picture of localised peritonitis, and were treated by percutaneous ultrasound guided drainage. Two other patients developed generalised peritonitis and were reoperated. One patient developed intraperitoneal collections and was operated too. Two patients developed prolonged external biliary fistula. The others were treated conservatively.

Another complication is the accidental dislodgment of T-tube, following biliary leakage and biliary peritonitis, localised or generalised. We have had two patients who needed urgent operation with reinsertion of t-tube. Another patient was reoperated for hemoperitoneum. Furthermore, the presence of T-tube in situ contributes to delayed return to normal activity and work and may cause patients persistent pain and discomfort [16].

Retained biliary stones were seen in $4(8.3 \%)$ patients, one following an open cholecystectomy and three other patients following laparoscopic cholecystectomy. All of them were treated by endoscopy.

Five patients developed bacteremia (10.41\%). Two of these had positive blood cultures, for the others it was not possible to do the blood culture, but manifested with rigors and high fever.

Other general complications (wound infection, pulmonary thromboembolism, internal hemorrhage) were found.

Another important limitation of this study is the impossibility of long term complications evaluation such as biliary strictures.

In conclusion this study has demonstrated a high incidence of biliary specific complications related T-tube use. As most surgeons still favour T-tube drainage it is probable that this practice should be re-assessed and embrace minimally invasive surgical techniques. 


\section{References:}

1. Glenn F. The physiological basis for surgical treatment of nonmalignant disease of the biliary tract. Surg Clin North Am 1958; 38: 471-85.

2. Keighley MRB, Burdon DW, Baddeley RM et al. Complications of supraduodenal choledochotomy: comparison of three methods of management. Br J Surg $1976 ; 63: 754-8$

3. Thornton JK. Observations on additional cases illustrating hepatic surgery. Lancet 1891;i:763-4

4. Apalakis A. An experimental evaluation of the types ofmaterial used for bile duct drainage tubes. BrJ Surg 1976; 63: 440-5.

5. Pitt HA, Postier RG, Cameron JL. Postoperative T-Tube cholangiography. Ann Surg 1980; 191:30-4.

6. Coppola R, Riccioni ME, Ciletti S, Cosentino L, Coco C, Magistrelli P, Picciocchi A(1997) Analysis of complications of endoscopic sphincterotomy for biliary stones in a consecutive series of 546 patients. Surg Endosc 11:129-132

7. Kim EK, Lee SK (2004) Laparoscopic treatment of choledocholithiasis using modified biliary stents. Surg Endosc 18:303-306

8. Griniatsos J, Karvounis E, Arbuckle J, Isla AM (2005) Costeffective method for laparoscopic choledochotomy. ANZ J Surg 75:35-3

9. Tang CN, Tai CK, Ha JP, Tsui KK, Wong DC, Li MK (2006) Antegrade biliary stenting versus T-tube drainage after laparoscopic choledochotomy. A comparative cohort study. Hepatogastroenterology 53:330-334
10. Apalakis A. An experimental evaluation of the types ofmaterial used for bile duct drainage tubes. BrJ Surg 1976; 63: $440-5$.

11. Leida Z, Ping B, Shuguang W, Yu H (2008) A randomized comparison of primary closure and T-tube drainage of the common bile duct after laparoscopic choledochotomy. Surg Endosc 22:1595-1600

12. Patelin JB (2003) Laparoscopic common bile duct exploration. Lessons learned from [12 years' experience. Surg Endosc 17:1705-1715

13. Paganini AM, Guerrieri M, Sarnari J, De Sanctis A, D'Ambrosio G, Lezoche G, Perretta S, Lezoche E (2007) Thirteen years' experience with laparoscopic transcystic common bile duct exploration for stones. Effectiveness and long-term results. Surg Endosc 21:34-40

14. JACOBS L. K., SHAYANI V., SACKIER J. M. Common bile duct T-tubes. A caveat and recommendations for management. Surg Endosc, 1998, 12: 60-2. 8.

15. MAURI A., CIRIGLIANO W. D., ZANON C., ALLUMINIO P., GIOANETTI N. R., MODO D. Choleperitoneum following Kehr's tube removal: conservative treatment with culdocentesis associated with sphincterotomy and endoscopic drainage of the bile ducts. Minerva Chir, 1991, 46: 279-81.

16. NOMURA T., NIKKUNI K., KATO H., YOSHIKAWA T., SASAKI K., SHIRAI Y., Hatakeyama K. Bile peritonitis due to a spontaneous perforation of the common bile duct: report of a case. Surg Today, 1996, 26: 822-4. 